\title{
THE SPECTRAL DENSITY OF THE SCATTERING MATRIX FOR HIGH ENERGIES
}

\author{
DANIEL BULGER AND ALEXANDER PUSHNITSKI
}

\begin{abstract}
We determine the density of eigenvalues of the scattering matrix of the Schrödinger operator with a short range potential in the high energy asymptotic regime. We give an explicit formula for this density in terms of the X-ray transform of the potential.
\end{abstract}

\section{Introduction and Main Result}

1.1. Introduction. The object of study in this paper is the (on-shell) scattering matrix $S(\lambda)$ corresponding to the scattering of a $d$-dimensional quantum particle on an external short range potential $V$ at the energy $\lambda>0$. The scattering matrix $S(\lambda)$ is a unitary operator in $L^{2}\left(\mathbb{S}^{d-1}\right)$ and the difference $S(\lambda)-I$ is compact. Thus, the eigenvalues of $S(\lambda)$ can be written as

$$
\exp \left(i \theta_{n}(\lambda)\right), \quad \theta_{n}(\lambda) \in[-\pi, \pi), \quad n \in \mathbb{N}
$$

and $\theta_{n}(\lambda) \rightarrow 0$ as $n \rightarrow \infty$. The quantities $\theta_{n}(\lambda)$ are known as scattering phases. The scattering phases are usually discussed in physics literature (see e.g. [6, Section 123]) under the additional assumption of the spherical symmetry of $V$; here we do not need this assumption.

Our aim is to study the asymptotic distribution of scattering phases $\left\{\theta_{n}(\lambda)\right\}_{n=1}^{\infty}$ when $\lambda \rightarrow \infty$. It turns out that after an appropriate scaling the asymptotic density of scattering phases can be described by a simple explicit formula involving the X-ray transform (see (1.5)) of the potential $V$. This formula has a semiclassical nature.

The key idea of this paper goes back to the work of M. Sh. Birman and D. R. Yafaev [1] (see also [2]), where the asymptotics of $\theta_{n}(\lambda)$ for a fixed $\lambda$ and $n \rightarrow \infty$ was determined for some class of potentials $V$. This asymptotic behaviour is not uniform in $\lambda$, and thus our results cannot be derived from those of [1]. However, both the results of [1] and our results are based on the following observation. The leading term of the asymptotics of $\theta_{n}(\lambda)$ (in both asymptotic regimes) is determined by the Born approximation of the scattering matrix. The Born approximation is essentially a pseudodifferential operator ( $\Psi \mathrm{DO})$ on the sphere $\mathbb{S}^{d-1}$ with the symbol given by the X-ray transform of $V$. Standard $\Psi D O$ results can be used to give spectral asymptotics for operators of such type. In both asymptotic regimes, the desired spectral asymptotics are given by a Weyl type formula involving the symbol of the $\Psi D O$.

1.2. The scattering matrix. Let us briefly recall the relevant definitions. Let $H_{0}=-\Delta$ and $H=-\Delta+V$ be the Schrödinger operators in $L^{2}\left(\mathbb{R}^{d}\right), d \geq 2$, where $V$ is the operator of

2010 Mathematics Subject Classification. Primary 35P25; Secondary 35P20, 81 U20.

Key words and phrases. Scattering matrix, Born approximation, eigenvalue distribution, X-ray transform. 
multiplication by a real-valued potential $V \in C\left(\mathbb{R}^{d}\right)$ which is assumed to satisfy the estimate

$$
|V(x)| \leq \frac{C}{(1+|x|)^{\rho}}, \quad \rho>1
$$

with some constant $C>0$. It is one of the fundamental facts of scattering theory [4, 5] that under the assumption (1.2) the wave operators

$$
W_{ \pm}=\operatorname{sslim}_{t \rightarrow \pm \infty} e^{i t H} e^{-i t H_{0}}
$$

exist and are complete; the scattering operator $\mathbf{S}=W_{+}^{*} W_{-}$is unitary in $L^{2}\left(\mathbb{R}^{d}\right)$ and commutes with $H_{0}$. Let $F: L^{2}\left(\mathbb{R}^{d}\right) \rightarrow L^{2}\left((0, \infty) ; L^{2}\left(\mathbb{S}^{d-1}\right)\right)$ be the unitary operator

$$
(F u)(\lambda, \omega)=\frac{1}{\sqrt{2}} \lambda^{(d-2) / 4} \widehat{u}(\sqrt{\lambda} \omega), \quad \lambda>0, \quad \omega \in \mathbb{S}^{d-1},
$$

where $\widehat{u}$ is the usual (unitary) Fourier transform of $u$. The operator $F$ diagonalises $H_{0}$, i.e.

$$
\left(F H_{0} u\right)(\lambda, \omega)=\lambda(F u)(\lambda, \omega), \quad \forall u \in C_{0}^{\infty}\left(\mathbb{R}^{d}\right) .
$$

The commutation relation $\mathbf{S} H_{0}=H_{0} \mathbf{S}$ implies that $F$ also diagonalises $\mathbf{S}$, i.e.

$$
(F \mathbf{S} u)(\lambda, \cdot)=S(\lambda)(F u)(\lambda, \cdot),
$$

where $S(\lambda): L^{2}\left(\mathbb{S}^{d-1}\right) \rightarrow L^{2}\left(\mathbb{S}^{d-1}\right)$ is the unitary operator known as the (on-shell) scattering matrix. See e.g. the book [9] for the details.

Under the assumption (1.2) the operator $S(\lambda)-I$ is compact and consequently the eigenvalues of $S(\lambda)$ (enumerated with multiplicities taken into account) can be written as (1.1) with $\theta_{n}(\lambda) \rightarrow 0$ as $n \rightarrow \infty$.

1.3. The purpose of the paper. Our purpose is to describe the asymptotic density of the eigenvalues of $S(\lambda)$ as $\lambda \rightarrow \infty$. We recall the estimate (see e.g. [9, Section 8.1])

$$
\|S(\lambda)-I\|=O\left(\lambda^{-1 / 2}\right), \quad \lambda \rightarrow \infty .
$$

This estimate is sharp, i.e. $O\left(\lambda^{-1 / 2}\right)$ cannot be replaced by $o\left(\lambda^{-1 / 2}\right)$; this can be seen by considering the case of a spherically symmetric potential and using the separation of variables. Thus, the spectrum of the scattering matrix $S(\lambda)$ for large $\lambda$ consists of a cluster of eigenvalues located on an arc of length $O\left(\lambda^{-1 / 2}\right)$ around 1. Let us define the eigenvalue counting measure for $S(\lambda)$. The estimate (1.3) suggests the following scaling: for $\lambda \geq 1$, set $k=\sqrt{\lambda}>0$ and define (using notation (1.1))

$$
\mu_{k}([\alpha, \beta])=\#\left\{n \in \mathbb{N}: \alpha \leq k \theta_{n}\left(k^{2}\right) \leq \beta\right\}, \quad[\alpha, \beta] \subset \mathbb{R} \backslash\{0\}
$$

where \# denotes the number of elements in the set. We will study the weak asymptotics of $\mu_{k}$ as $k \rightarrow \infty$, i.e. we consider the asymptotics of the integrals

$$
\int_{-\infty}^{\infty} \psi(t) \mathrm{d} \mu_{k}(t), \quad k \rightarrow \infty
$$

for test functions $\psi \in C_{0}^{\infty}(\mathbb{R} \backslash\{0\})$. 
1.4. Main result. In order to describe the weak limit of the measures $\mu_{k}$, we need to fix some notation. For any $\omega \in \mathbb{S}^{d-1}$, let $\Lambda_{\omega} \subset \mathbb{R}^{d}$ denote the hyperplane passing through the origin and orthogonal to $\omega$. We equip both $\mathbb{S}^{d-1}$ and $\Lambda_{\omega}$ with the standard $(d-1)$-dimensional Lebesgue measure (=Euclidean area). We set

$$
X(\omega, \eta)=-\frac{1}{2} \int_{-\infty}^{\infty} V(t \omega+\eta) \mathrm{d} t, \quad \omega \in \mathbb{S}^{d-1}, \quad \eta \in \Lambda_{\omega} .
$$

The function $X$ (up to a multiplicative factor) is known as the X-ray transform of $V$ in the inverse problem literature. The following elementary estimate is a direct consequence of (1.2):

$$
|X(\omega, \eta)| \leq C(V)(1+|\eta|)^{1-\rho}, \quad \omega \in \mathbb{S}^{d-1}, \quad \eta \in \Lambda_{\omega}
$$

with some constant $C(V)$. We define a measure $\mu$ on $\mathbb{R} \backslash\{0\}$ by

$$
\mu([\alpha, \beta])=(2 \pi)^{1-d} \operatorname{meas}\left\{(\omega, \eta) \in \mathbb{S}^{d-1} \times \Lambda_{\omega}: \alpha \leq X(\omega, \eta) \leq \beta\right\}, \quad[\alpha, \beta] \subset \mathbb{R} \backslash\{0\},
$$

where meas denotes the usual product measure. By the boundedness of $V$, the measure $\mu$ has a compact support. The measure $\mu$ need not be absolutely continuous. The measure $\mu$ may be weakly singular at zero in the following sense: $\mu((0, \infty))$ or $\mu((-\infty, 0))$ may be infinite, but, by the estimate (1.6) we have

$$
\int_{-\infty}^{\infty}|t|^{\ell} \mathrm{d} \mu(t)<\infty, \quad \forall \ell>(d-1) /(\rho-1) .
$$

Our main result is as follows:

Theorem 1.1. Let $V \in C\left(\mathbb{R}^{d}\right)$ be a potential satisfying (1.2). Then for any test function $\psi \in C_{0}^{\infty}(\mathbb{R} \backslash\{0\})$,

$$
\lim _{k \rightarrow \infty} k^{1-d} \int_{-\infty}^{\infty} \psi(t) \mathrm{d} \mu_{k}(t)=\int_{-\infty}^{\infty} \psi(t) \mathrm{d} \mu(t)
$$

This can be more succinctly put as the weak convergence of the measures

$$
k^{1-d} \mu_{k} \rightarrow \mu, \quad k \rightarrow \infty .
$$

Much of the inspiration for both the content of this paper and the proofs may be found in [7], where similar asymptotics are determined for the spectrum of the Landau Hamiltonian (i.e. two-dimensional Schrödinger operator with a constant homogeneous magnetic field) perturbed by a potential which obeys the same condition (1.2).

We would like to mention also the paper [10] where the high energy asymptotic distribution of the phases $\theta_{n}(\lambda)$ was studied for scattering problems on manifolds of a certain special class. The results of [10] are much more detailed than ours and include the asymptotics of the pair correlation measure.

1.5. Comparison with [1]. In [1], the case of potentials with the power asymptotics at infinity of the type

$$
V(x)=v(x /|x|)|x|^{-\rho}(1+o(1)), \quad|x| \rightarrow \infty, \quad \rho>1,
$$

was considered. Using our notation $\mu_{k}, \mu$, the result of [1] can be written as

$$
\begin{aligned}
k^{1-d} \mu_{k}((\varepsilon, \infty)) & \sim \mu((\varepsilon, \infty)), \\
k^{1-d} \mu_{k}((-\infty,-\varepsilon)) & \sim \mu((-\infty,-\varepsilon)),
\end{aligned}
$$


when $k>0$ is fixed and $\varepsilon \rightarrow+0$. Here $a \sim b$ means $\frac{a}{b} \rightarrow 1$.

Clearly, our main result (1.9) is expressed by the same formula as (1.11), but the asymptotic regimes are different. Neither of the results (1.9) , (1.11) implies the other one.

1.6. Semiclassical interpretation. By the definition of the scattering operator $\mathbf{S}$, for any $\psi \in L^{2}\left(\mathbb{R}^{d}\right)$ we have

$$
\begin{aligned}
i((\mathbf{S}-I) \psi, \psi) & =i \lim _{t \rightarrow \infty}\left(\left(e^{-2 i t H} e^{i t H_{0}} \psi, e^{-i t H_{0}} \psi\right)-\|\psi\|^{2}\right)=i \int_{0}^{\infty} \frac{\mathrm{d}}{\mathrm{d} t}\left(e^{-2 i t H} e^{i t H_{0}} \psi, e^{-i t H_{0}} \psi\right) \mathrm{d} t \\
& =\int_{0}^{\infty}\left(V e^{-2 i t H} e^{i t H_{0}} \psi, e^{-i t H_{0}} \psi\right) \mathrm{d} t+\int_{0}^{\infty}\left(V e^{i t H_{0}} \psi, e^{2 i t H} e^{-i t H_{0}} \psi\right) \mathrm{d} t .
\end{aligned}
$$

If $\psi$ corresponds to large energies, the right hand side can be approximated by the first term in its expansion in powers of $V$. This means that we can replace $e^{i t H}$ by $e^{i t H_{0}}$ in the above expressions, and so

$$
i((\mathbf{S}-I) \psi, \psi) \approx \int_{-\infty}^{\infty}\left(V e^{-i t H_{0}} \psi, e^{-i t H_{0}} \psi\right) \mathrm{d} t, \quad \psi \in L^{2}\left(\mathbb{R}^{d}\right),
$$

which is exactly the Born approximation in the time-dependent picture.

In order to write down the classical analogue of the right hand side of (1.12), assume that $\psi$ is concentrated near $x$ in the coordinate representation and near $p$ in the momentum representation. Then $\psi$ represents a particle with the coordinate $x$ and momentum $p$, and in the same way $e^{-i t H_{0}} \psi$ represents a particle with the coordinate $x+2 p t$ and momentum $p$. Thus, the classical analogue of the right hand side of (1.12) is

$$
\int_{-\infty}^{\infty} V(x+2 p t) \mathrm{d} t=\frac{1}{2|p|} \int_{-\infty}^{\infty} V\left(x+\omega t^{\prime}\right) \mathrm{d} t^{\prime}, \quad(x, p) \in \mathbb{R}^{d} \times \mathbb{R}^{d},
$$

where $\omega=\frac{p}{|p|} \in \mathbb{S}^{d-1}$. This calculation explains the appearance of the X-ray transform in the asymptotics of $S(\lambda)$.

1.7. Key steps of the proof. First we recall the stationary representation for the scattering matrix. For $k>0$ and $\rho>1$, we define the operator $\Gamma_{\rho}(k): L^{2}\left(\mathbb{R}^{d}\right) \rightarrow L^{2}\left(\mathbb{S}^{d-1}\right)$ by

$$
\left(\Gamma_{\rho}(k) u\right)(\omega)=\frac{1}{\sqrt{2}} k^{(d-2) / 2}(2 \pi)^{-d / 2} \int_{\mathbb{R}^{d}} u(x)\langle x\rangle^{-\rho / 2} e^{-i k\langle x, \omega\rangle} \mathrm{d} x, \quad \omega \in \mathbb{S}^{d-1},
$$

where $\langle x\rangle=\left(1+|x|^{2}\right)^{1 / 2}$. By the Sobolev trace theorem, $\Gamma_{\rho}(k)$ is bounded for $\rho>1$. Next, let

$$
T(z)=\langle x\rangle^{-\rho / 2}(H-z I)^{-1}\langle x\rangle^{-\rho / 2}, \quad \operatorname{Im} z>0
$$

according to the limiting absorption principle, the limits $T\left(k^{2} \pm i 0\right)$ exist in the operator norm for all $k>0$. Denote by $J$ the bounded operator of multiplication by $\langle x\rangle^{\rho} V(x)$ in $L^{2}\left(\mathbb{R}^{d}\right)$. The stationary representation for the scattering matrix can be written as (see e.g.[9], Section 6.6])

$$
S\left(k^{2}\right)=I-2 \pi i \Gamma_{\rho}(k)\left(J-J T\left(k^{2}+i 0\right) J\right) \Gamma_{\rho}(k)^{*}, \quad k>0 .
$$

The asymptotic density of eigenvalues of $S\left(k^{2}\right)$ for large $k$ is determined by the Born approximation of the scattering matrix, defined as

$$
S_{B}\left(k^{2}\right)=I-2 \pi i \Gamma_{\rho}(k) J \Gamma_{\rho}(k)^{*}, \quad k>0 .
$$


The key observation due to M. Birman and D. Yafaev [1] is that the operator $\Gamma_{\rho}(k) J \Gamma_{\rho}(k)^{*}$ in $L^{2}\left(\mathbb{S}^{d-1}\right)$ with the integral kernel

$$
2^{-1} k^{d-2}(2 \pi)^{-d} \int_{\mathbb{R}^{d}} e^{-i k\left\langle\omega-\omega^{\prime}, x\right\rangle} V(x) \mathrm{d} x, \quad \omega, \omega^{\prime} \in \mathbb{S}^{d-1}
$$

can be represented as a $\Psi D O$ on the sphere with the symbol (up to inessential constants) $X(\omega, \eta)$. We combine this observation with the standard semiclassical pseudodifferential techniques to obtain the spectral asymptotics of $S_{B}\left(k^{2}\right)$. In this way we prove the asymptotic formula (see Lemma 3.2)

$$
\lim _{k \rightarrow \infty} k^{1-d} \operatorname{Tr}\left(\operatorname{Im} k S_{B}\left(k^{2}\right)\right)^{\ell}=\int_{-\infty}^{\infty} t^{\ell} \mathrm{d} \mu(t)
$$

for any natural number $\ell>(d-1) /(\rho-1)$; note that the r.h.s. of (1.15) is finite by (1.7).

Using (1.15) and the estimates for the Schatten norm of $S\left(k^{2}\right)-S_{B}\left(k^{2}\right)$ we prove that (1.8) holds true for test functions $\psi(t)$ which coincide with $t^{\ell}$ for all sufficiently small $t$. Theorem 1.1 follows by an application of the Weierstrass approximation theorem.

1.8. Acknowledgements. The authors are grateful to Y. Safarov for expert advice concerning the $\Psi$ DO aspect of this work. The authors are grateful to N. Filonov and D. Yafaev for a number of useful discussions and remarks on the text of the paper.

\section{Preliminary statements}

2.1. The limiting absorption principle and its consequences. First we need some notation. We denote by $S_{p}, p \geq 1$, the usual Schatten class and by $\|\cdot\|_{p}$ the associated norm. Let $X_{\rho}$ be the normed linear space of all potentials $V \in C\left(\mathbb{R}^{d}\right)$ satisfying (1.2) with the norm

$$
\|V\|_{X_{\rho}}=\sup _{x \in \mathbb{R}^{d}}|V(x)|\langle x\rangle^{\rho} .
$$

We recall the following estimates from [9]:

Proposition 2.1. Let $V \in X_{\rho}$ with some $\rho>1$. Then for any $\ell \geq 1$ satisfying $\ell>\frac{d-1}{\rho-1}$, one has

$$
\begin{aligned}
& \sup _{k \geq 1} k^{\frac{1-d}{\ell}}\left\|k \operatorname{Im} S_{B}\left(k^{2}\right)\right\|_{\ell} \leq C(\ell, \rho, d)\|V\|_{X_{\rho}}, \\
& \sup _{k \geq 1} k^{\frac{1-d}{\ell}}\left\|k^{2} \operatorname{Im}\left(S_{B}\left(k^{2}\right)-S\left(k^{2}\right)\right)\right\|_{\ell} \leq C(\ell, \rho, d, V), \\
& \sup _{k \geq 1} k^{\frac{1-d}{\ell}}\left\|k \operatorname{Im} S\left(k^{2}\right)\right\|_{\ell} \leq C(\ell, \rho, d, V) .
\end{aligned}
$$

The estimate (2.1) is a direct consequence of [9, Proposition 8.1.3]. The estimate (2.2) is proven in [9, Proposition 8.1.4]. The estimate (2.3) is a direct consequence of (2.1) and (2.2).

Lemma 2.2. Let $V \in X_{\rho}$ with $\rho>1$. Then for any integer $\ell \geq 1$ satisfying $\ell>\frac{d-1}{\rho-1}$, one has

$$
\left|\operatorname{Tr}\left(k \operatorname{Im} S\left(k^{2}\right)\right)^{\ell}-\operatorname{Tr}\left(k \operatorname{Im} S_{B}\left(k^{2}\right)\right)^{\ell}\right|=O\left(k^{d-2}\right), \quad k \rightarrow \infty
$$


Proof. From

$$
A^{\ell}-B^{\ell}=\sum_{j=0}^{\ell-1} A^{j}(A-B) B^{\ell-1-j}
$$

one easily obtains

$$
\left|\operatorname{Tr}\left(A^{\ell}-B^{\ell}\right)\right| \leq \ell\|A-B\|_{\ell} \max \left\{\|A\|_{\ell}^{\ell-1},\|B\|_{\ell}^{\ell-1}\right\}, \quad A, B \in S_{\ell} .
$$

Thus, it suffices to prove the relation

$$
\left\|k \operatorname{Im}\left(S\left(k^{2}\right)-S_{B}\left(k^{2}\right)\right)\right\|_{\ell} \max \left\{\left\|k \operatorname{Im} S\left(k^{2}\right)\right\|_{\ell}^{\ell-1},\left\|k \operatorname{Im} S_{B}\left(k^{2}\right)\right\|_{\ell}^{\ell-1}\right\}=O\left(k^{d-2}\right), \quad k \rightarrow \infty .
$$

The latter relation follows by combining (2.1)-(2.3).

2.2. Semiclassical $\Psi D O$ on the sphere. A semiclassical $\Psi D O$ in $L^{2}\left(\mathbb{S}^{d-1}\right)$ can be defined in a variety of ways; below we describe a slightly non-standard approach to this definition, which will simplify our exposition in Section 3.

For $\omega, \omega^{\prime} \in \mathbb{S}^{d-1}$ such that $\omega+\omega^{\prime} \neq 0$, we set

$$
\kappa=\kappa\left(\omega, \omega^{\prime}\right)=\frac{\omega+\omega^{\prime}}{\left|\omega+\omega^{\prime}\right|} \in \mathbb{S}^{d-1} .
$$

Clearly, $\kappa\left(\omega, \omega^{\prime}\right)$ is a smooth function of $\left(\omega, \omega^{\prime}\right) \in \mathbb{S}^{d-1} \times \mathbb{S}^{d-1}$ away from the anti-diagonal

$$
\mathrm{AD}=\left\{\left(\omega, \omega^{\prime}\right) \in \mathbb{S}^{d-1} \times \mathbb{S}^{d-1} \mid \omega+\omega^{\prime}=0\right\} .
$$

In order to overcome the (inessential) difficulties related to the singularity of $\kappa$ at the antidiagonal, we will assume that our amplitudes vanish in an open neighbourhood of AD. We will say that a function $b=b\left(\omega, \omega^{\prime}, \eta\right), \omega, \omega^{\prime} \in \mathbb{S}^{d-1}, \eta \in \Lambda_{\kappa\left(\omega, \omega^{\prime}\right)}$, is an admissible amplitude, if:

- $b$ is a $C^{\infty}$-smooth function of its arguments;

- $b\left(\omega, \omega^{\prime}, \eta\right)=0$ if $|\eta|$ is sufficiently large;

- $b\left(\omega, \omega^{\prime}, \eta\right)=0$ if $\left(\omega, \omega^{\prime}\right)$ are in an open neighbourhood of AD.

For an admissible amplitude $b$ and a semiclassical parameter $k>0$, we define the operator $\mathrm{Op}_{k}[b]$ in $L^{2}\left(\mathbb{S}^{d-1}\right)$ via its integral kernel

$$
\mathrm{Op}_{k}[b]\left(\omega, \omega^{\prime}\right)=\left(\frac{k}{2 \pi}\right)^{d-1} \int_{\Lambda_{\kappa}\left(\omega, \omega^{\prime}\right)} e^{-i k\left\langle\omega-\omega^{\prime}, \eta\right\rangle} b\left(\omega, \omega^{\prime}, \eta\right) \mathrm{d} \eta,
$$

where $\omega, \omega^{\prime} \in \mathbb{S}^{d-1}$. It is easy to see that for $\omega \neq \omega^{\prime}$ one has

$$
\mathrm{Op}_{k}[b]\left(\omega, \omega^{\prime}\right)=O\left(k^{-\infty}\right), \quad k \rightarrow \infty .
$$

This shows that the values of the amplitude $b\left(\omega, \omega^{\prime}, \eta\right)$ away from an open neighbourhood of the diagonal $\omega=\omega^{\prime}$ do not affect the asymptotic properties of the operator $\mathrm{Op}_{k}[b]$ as $k \rightarrow \infty$.

Proposition 2.3. For any admissible amplitude $b$ and any $k>0$, the operator $\mathrm{Op}_{k}[b]$ is trace class, and for any $\ell \in \mathbb{N}$ one has

$$
\lim _{k \rightarrow \infty}\left(\frac{k}{2 \pi}\right)^{-d+1} \operatorname{Tr}\left(\mathrm{Op}_{k}[b]\right)^{\ell}=\int_{\mathbb{S}^{d-1}} \int_{\Lambda_{\omega}} b(\omega, \omega, \eta)^{\ell} \mathrm{d} \eta \mathrm{d} \omega .
$$


Sketch of proof. The proof follows standard methods of $\Psi$ DO theory; see e.g. [3, Theorem 9.6] for a similar statement in the context of operators in $\mathbb{R}^{n}$. Here we only outline the main steps.

First note that for $\ell=1$ the result of Proposition 2.3 is trivial, since by a direct evaluation of trace we have the identity

$$
\left(\frac{k}{2 \pi}\right)^{-d+1} \operatorname{Tr}\left(\mathrm{Op}_{k}[b]\right)=\int_{\mathbb{S}^{d-1}} \int_{\Lambda_{\omega}} b(\omega, \omega, \eta) \mathrm{d} \eta \mathrm{d} \omega .
$$

Next, using the local coordinates on the sphere and the composition formula for symbols of $\Psi D O s$ (see e.g. [3, Proposition 7.7]), we obtain the following statement. For any $N>0$ there exists $M>0$ such that $\left(\mathrm{Op}_{k}[b]\right)^{\ell}$ can be represented as

$$
\left(\mathrm{Op}_{k}[b]\right)^{\ell}=\sum_{m=0}^{M} k^{-m} \mathrm{Op}_{k}\left[b_{m}\right]+R_{k}
$$

where $b_{m}$ are admissible symbols, $b_{0}$ is such that

$$
b_{0}(\omega, \omega, \eta)=b(\omega, \omega, \eta)^{\ell}, \quad \forall \omega \in \mathbb{S}^{d-1}, \quad \forall \eta \in \Lambda_{\omega},
$$

and $R_{k}$ is an integral operator with a smooth kernel which satisfies

$$
\sup _{\omega, \omega^{\prime}}\left|R_{k}\left(\omega, \omega^{\prime}\right)\right|=O\left(k^{-N}\right), \quad k \rightarrow \infty .
$$

Now taking $N>d-1$ and evaluating the trace in (2.8), we get

$$
\lim _{k \rightarrow \infty}\left(\frac{k}{2 \pi}\right)^{-d+1} \operatorname{Tr}\left(\mathrm{Op}_{k}[b]\right)^{\ell}=\lim _{k \rightarrow \infty}\left(\frac{k}{2 \pi}\right)^{-d+1} \operatorname{Tr}\left(\mathrm{Op}_{k}\left[b_{0}\right]\right)=\int_{\mathbb{S}^{d-1}} \int_{\Lambda_{\omega}} b_{0}(\omega, \omega, \eta) \mathrm{d} \eta \mathrm{d} \omega .
$$

In view of (2.9), this proves the required identity.

\section{Proof of Theorem 1.1}

3.1. The Born approximation with $V \in C_{0}^{\infty}\left(\mathbb{R}^{d}\right)$.

Lemma 3.1. Let $V \in C_{0}^{\infty}\left(\mathbb{R}^{d}\right)$. Then for any $\ell \in \mathbb{N}$, one has

$$
\lim _{k \rightarrow \infty} k^{1-d} \operatorname{Tr}\left(k \operatorname{Im} S_{B}\left(k^{2}\right)\right)^{\ell}=\int_{-\infty}^{\infty} t^{\ell} d \mu(t) .
$$

Proof. 1. For ease of notation we write $Q(k)=k \operatorname{Im} S_{B}\left(k^{2}\right)$. By (1.13) and (1.14), $Q(k)$ is the integral operator in $L^{2}\left(\mathbb{S}^{d-1}\right)$ with the integral kernel

$$
Q(k)\left(\omega, \omega^{\prime}\right)=-\frac{1}{2}\left(\frac{k}{2 \pi}\right)^{d-1} \int_{\mathbb{R}^{d}} e^{-i k\left\langle\omega-\omega^{\prime}, x\right\rangle} V(x) \mathrm{d} x, \quad \omega, \omega^{\prime} \in \mathbb{S}^{d-1} .
$$

For fixed $\omega, \omega^{\prime}, \omega+\omega^{\prime} \neq 0$, let $\kappa=\kappa\left(\omega, \omega^{\prime}\right)$ be as in (2.5). Write any $x \in \mathbb{R}^{d}$ as $x=\kappa t+\eta$ with $t \in \mathbb{R}, \eta \in \Lambda_{\kappa}$. Note that by the orthogonality relation $\left(\omega-\omega^{\prime}\right) \perp \kappa$, one has

$$
\left\langle\omega-\omega^{\prime}, x\right\rangle=\left\langle\omega-\omega^{\prime}, \eta\right\rangle \text {. }
$$


Thus, the integral kernel of $Q(k)$ can be rewritten as

$$
\begin{aligned}
Q(k)\left(\omega, \omega^{\prime}\right)=-\frac{1}{2}\left(\frac{k}{2 \pi}\right)^{d-1} \int_{\Lambda_{\kappa\left(\omega, \omega^{\prime}\right)}} & \int_{-\infty}^{\infty} e^{-i k\left\langle\omega-\omega^{\prime}, \eta\right\rangle} V\left(\kappa\left(\omega, \omega^{\prime}\right) t+\eta\right) \mathrm{d} t \mathrm{~d} \eta \\
& =\left(\frac{k}{2 \pi}\right)^{d-1} \int_{\Lambda_{\kappa\left(\omega, \omega^{\prime}\right)}} e^{-i k\left\langle\omega-\omega^{\prime}, \eta\right\rangle} X\left(\kappa\left(\omega, \omega^{\prime}\right), \eta\right) \mathrm{d} \eta
\end{aligned}
$$

where $X$ is given by (1.5). From here we directly obtain the required identity (3.1) in the case $\ell=1$ by integrating the kernel of $Q(k)$ over the diagonal. Now it remains to prove (3.1) for $\ell \geq 2$.

2. Let $\chi_{0} \in C_{0}^{\infty}\left(\mathbb{S}^{d-1} \times \mathbb{S}^{d-1}\right)$ be such that $\chi_{0}\left(\omega, \omega^{\prime}\right)=1$ in an open neighbourhood of the diagonal $\omega=\omega^{\prime}$ and $\chi_{0}\left(\omega, \omega^{\prime}\right)=0$ in an open neighbourhood of the anti-diagonal $\omega+\omega^{\prime}=0$. Denote $\chi_{1}=1-\chi_{0}$, and let

$$
Q(k)=Q_{0}(k)+Q_{1}(k)
$$

where $Q_{j}(k)$ is the operator with the integral kernel $\chi_{j}\left(\omega, \omega^{\prime}\right) Q(k)\left(\omega, \omega^{\prime}\right)$. By the fast decay of the Fourier transform of $V$ and by the fact that $\left|\omega-\omega^{\prime}\right|$ is separated away from zero on the support of $\chi_{1}$, we see that

$$
\sup _{\omega, \omega^{\prime}}\left|Q_{1}(k)\left(\omega, \omega^{\prime}\right)\right|=O\left(k^{-\infty}\right), \quad k \rightarrow \infty .
$$

Thus, it suffices to prove that

$$
\lim _{k \rightarrow \infty} k^{1-d} \operatorname{Tr}\left(Q_{0}(k)\right)^{\ell}=\int_{-\infty}^{\infty} t^{\ell} d \mu(t) .
$$

From (3.2) it follows that $Q_{0}(k)$ can be represented as a semiclassical $\Psi$ DO on the sphere of the type (2.6):

$$
Q_{0}(k)=\mathrm{Op}_{k}[b], \quad \text { where } \quad b\left(\omega, \omega^{\prime}, \eta\right)=\chi_{0}\left(\omega, \omega^{\prime}\right) X\left(\kappa\left(\omega, \omega^{\prime}\right), \eta\right),
$$

$b$ is an admissible amplitude in the sense discussed in Section 2.2, and $X$ is given by (1.5). Applying Proposition 2.3, we get

$$
\lim _{k \rightarrow \infty} k^{1-d} \operatorname{Tr}\left(Q_{0}(k)\right)^{\ell}=(2 \pi)^{1-d} \int_{\mathbb{S}^{d-1}} \int_{\Lambda_{\omega}} X(\omega, \eta)^{\ell} \mathrm{d} \eta \mathrm{d} \omega=\int_{-\infty}^{\infty} t^{\ell} d \mu(t),
$$

as required.

\subsection{The Born approximation with general $V$.}

Lemma 3.2. Let $V \in X_{\rho}$ with $\rho>1$. Then for any integer $\ell \geq 1$ satisfying $\ell>\frac{d-1}{\rho-1}$ one has

$$
\lim _{k \rightarrow \infty} k^{1-d} \operatorname{Tr}\left(\operatorname{Im} k S_{B}\left(k^{2}\right)\right)^{\ell}=\int_{-\infty}^{\infty} t^{\ell} \mathrm{d} \mu(t) .
$$

Proof. Let $X_{\rho}^{0}$ be the closure of $C_{0}^{\infty}\left(\mathbb{R}^{d}\right)$ in $X_{\rho}$. For any $\ell>\frac{d-1}{\rho-1}$, denote

$$
\begin{aligned}
g_{\ell}(V) & =\int_{-\infty}^{\infty} t^{\ell} \mathrm{d} \mu(t), \\
g_{\ell}^{+}(V) & =\limsup _{k \rightarrow \infty} k^{1-d} \operatorname{Tr}\left(k \operatorname{Im} S_{B}\left(k^{2}\right)\right)^{\ell}, \\
g_{\ell}^{-}(V) & =\liminf _{k \rightarrow \infty} k^{1-d} \operatorname{Tr}\left(k \operatorname{Im} S_{B}\left(k^{2}\right)\right)^{\ell} .
\end{aligned}
$$


By Lemma 3.1, for all $V \in C_{0}^{\infty}\left(\mathbb{R}^{d}\right)$ we have

$$
g_{\ell}(V)=g_{\ell}^{+}(V)=g_{\ell}^{-}(V) .
$$

Recall that $S_{B}\left(k^{2}\right)$ depends linearly on $V$. Using this fact and the estimates (2.1) and (2.4), it is easy to check that $g_{\ell}^{ \pm}(V)$ are continuous functionals on $X_{\rho}$. Next, using the last equality in (3.3) and the estimate (1.6), it is easy to see that $g_{\ell}(V)$ is a continuous functional on $X_{\rho}$. Thus, by an approximation argument, (3.4) holds for any $V \in X_{\rho}^{0}$. Finally, for any $V \in X_{\rho}$ and a given $\ell>\frac{d-1}{\rho-1}$, choose $\rho_{1}$ such that $1<\rho_{1}<\rho$ with $\ell>\frac{d-1}{\rho_{1}-1}$. Then $X_{\rho} \subset X_{\rho_{1}}^{0}$ and the previous argument proves (3.4) for all $V \in X_{\rho_{1}}^{0}$ which suffices.

\subsection{From the Born approximation to the full scattering matrix.}

Lemma 3.3. Let $V \in X_{\rho}$ with $\rho>1$. Then for any integer $\ell \geq 1$ satisfying $\ell+2>\frac{d-1}{\rho-1}$,

$$
\lim _{k \rightarrow \infty} k^{1-d} \int_{-\infty}^{\infty} t^{\ell} \mathrm{d} \mu_{k}(t)=\int_{-\infty}^{\infty} t^{\ell} \mathrm{d} \mu(t) .
$$

Proof. By Lemmas 2.2 and 3.2, it suffices to prove

$$
\lim _{k \rightarrow \infty} k^{1-d}\left|\int_{-\infty}^{\infty} t^{\ell} \mathrm{d} \mu_{k}(t)-\operatorname{Tr}\left(\operatorname{Im} k S\left(k^{2}\right)\right)^{\ell}\right|=0 .
$$

Recalling the definition (1.4) of the measure $\mu_{k}$, one sees that (3.5) is equivalent to

$$
\lim _{k \rightarrow \infty} k^{1-d+\ell}\left|\sum_{n=1}^{\infty}\left[\left(\theta_{n}\left(k^{2}\right)\right)^{\ell}-\left(\sin \theta_{n}\left(k^{2}\right)\right)^{\ell}\right]\right|=0 .
$$

By (1.3) we have $0<\left|\theta_{n}\left(k^{2}\right)\right|<\pi / 4$ for all sufficiently large $k$ and all $n$. From the elementary estimates $\left|\theta_{n}\right| \leq 2\left|\sin \theta_{n}\right|$ and $\left|\theta_{n}-\sin \theta_{n}\right| \leq C\left|\sin \theta_{n}\right|^{3}$ which hold for $\left|\theta_{n}\right|<\pi / 4$, it follows that for all sufficiently large $k$

$$
\begin{gathered}
k^{1-d+\ell} \sum_{n=1}^{\infty}\left|\left(\theta_{n}\right)^{\ell}-\left(\sin \theta_{n}\right)^{\ell}\right| \leq k^{1-d+\ell} \sum_{n=1}^{\infty}\left(\left|\theta_{n}-\sin \theta_{n}\right| \sum_{j=0}^{\ell-1}\left|\theta_{n}\right|^{j}\left|\sin \theta_{n}\right|^{\ell-1-j}\right) \\
\leq k^{1-d+\ell} C(\ell) \sum_{n=1}^{\infty}\left|\sin \theta_{n}\right|^{\ell+2}=k^{1-d-2} C(\ell)\left\|\operatorname{Im} k S\left(k^{2}\right)\right\|_{\ell+2}^{\ell+2} .
\end{gathered}
$$

Now (3.6) follows by applying the estimate (2.3) for $\left\|\operatorname{Im} k S\left(k^{2}\right)\right\|_{\ell+2}$ to the result just obtained.

Proof of Theorem 1.1. By the estimate (1.3), the supports of $\mu_{k}$ are bounded uniformly in $k \geq 1$. On the other hand, by the boundedness of $V$, the support of $\mu$ is also bounded. Thus, we may choose $T>0$ such that

$$
\operatorname{supp} \mu \subset[-T, T] \text { and } \operatorname{supp} \mu_{k} \subset[-T, T] \quad \text { for all } k \geq 1 .
$$

Next, fix $\psi \in C_{0}^{\infty}(\mathbb{R} \backslash\{0\})$, and let $\ell_{0}$ be an even natural number satisfying $\ell_{0}>\frac{d-1}{\rho-1}$. Since $\psi(t)$ vanishes near $t=0$ by assumption, the function $\psi(t) / t^{\ell_{0}}$ is smooth. By the Weierstrass approximation theorem, for any $\varepsilon>0$ there exists a polynomial $\psi_{0}(t)$ such that

$$
\left|\psi(t) t^{-\ell_{0}}-\psi_{0}(t)\right| \leq \varepsilon, \quad \forall t \in[-T, T] .
$$


Denoting $\psi_{ \pm}(t)=\left(\psi_{0}(t) \pm \varepsilon\right) t^{\ell_{0}}$, we obtain

$$
\begin{aligned}
& \psi_{-}(t) \leq \psi(t) \leq \psi_{+}(t), \quad \forall t \in[-T, T], \\
& \psi_{+}(t)-\psi_{-}(t)=2 \varepsilon t^{\ell_{0}} .
\end{aligned}
$$

By (3.7), we get

$$
\int_{-\infty}^{\infty} \psi_{-}(t) \mathrm{d} \mu_{k}(t) \leq \int_{-\infty}^{\infty} \psi(t) \mathrm{d} \mu_{k}(t) \leq \int_{-\infty}^{\infty} \psi_{+}(t) \mathrm{d} \mu_{k}(t)
$$

By construction, $\psi_{ \pm}(t)$ are polynomials which involve powers $t^{m}$ with $m \geq \ell_{0}$. Thus, we can apply Lemma 3.3 to (3.9), which yields

$$
\begin{aligned}
& \limsup _{k \rightarrow \infty} k^{1-d} \int_{-\infty}^{\infty} \psi(t) \mathrm{d} \mu_{k}(t) \leq \int_{-\infty}^{\infty} \psi_{+}(t) \mathrm{d} \mu(t), \\
& \liminf _{k \rightarrow \infty} k^{1-d} \int_{-\infty}^{\infty} \psi(t) \mathrm{d} \mu_{k}(t) \geq \int_{-\infty}^{\infty} \psi_{-}(t) \mathrm{d} \mu(t) .
\end{aligned}
$$

On the other hand, by (3.7), (3.8),

$$
\int_{-\infty}^{\infty} \psi_{-}(t) \mathrm{d} \mu(t) \leq \int_{-\infty}^{\infty} \psi(t) \mathrm{d} \mu(t) \leq \int_{-\infty}^{\infty} \psi_{+}(t) \mathrm{d} \mu(t)
$$

and

$$
\int_{-\infty}^{\infty} \psi_{+}(t) \mathrm{d} \mu(t)-\int_{-\infty}^{\infty} \psi_{-}(t) \mathrm{d} \mu(t)=2 \varepsilon \int_{-\infty}^{\infty} t^{\ell_{0}} \mathrm{~d} \mu(t)
$$

By (1.6), the integral in the right hand side of the last estimate is finite; denote this integral by $C$. Combining the above estimates, we obtain

$$
\begin{gathered}
\limsup _{k \rightarrow \infty} k^{1-d} \int_{-\infty}^{\infty} \psi(t) \mathrm{d} \mu_{k}(t) \leq \int_{-\infty}^{\infty} \psi(t) \mathrm{d} \mu(t)+2 \varepsilon C, \\
\liminf _{k \rightarrow \infty} k^{1-d} \int_{-\infty}^{\infty} \psi(t) \mathrm{d} \mu_{k}(t) \geq \int_{-\infty}^{\infty} \psi(t) \mathrm{d} \mu(t)-2 \varepsilon C .
\end{gathered}
$$

Since $\varepsilon>0$ can be taken arbitrary small, we obtain the required statement.

\section{REFERENCES}

[1] M. Sh. Birman And D. R. Yafaev, Asymptotic behaviour of the spectrum of the scattering matrix, J. Sov. Math. 25 (1984), 793-814.

[2] M. Sh. Birman and D. R. YAfaev, Spectral properties of the scattering matrix, St Petersburg Math. Journal, 4, no. 6, (1993), 1055-1079.

[3] M. Dimassi And J. SJÖstrand, Spectral asymptotics in the semi-classical limit, Cambridge University Press, 1999.

[4] T. Kato, Wave operators and similarity for some non-selfadjoint operators, Math. Ann., 162, no. 2 (1965/1966), 258-279.

[5] S. T. KurodA, Some remarks on scattering theory for Schrödinger operators, J. Fac. Sci., Univ. Tokyo, ser. I, 17 (1970), 315-329.

[6] L. D. Landau, E. M. Lifschitz, Quantum mechanics: non-relativistic theory. Course of Theoretical Physics, Vol. 3, Addison-Wesley Series in Advanced Physics, Pergamon Press, 1958.

[7] A. Pushnitski, G. Raikov and C. Villegas-Blas, Asymptotic density of eigenvalue clusters for the perturbed Landau Hamiltonian, preprint 2011, arXiv:1110.3098v1

[8] M. A. Shubin, Pseudodifferential operators and spectral theory, Springer Verlag, 1987.

[9] D. YAfaev, Mathematical scattering theory. Analytic theory, American Mathematical Society, 2010. 
[10] S. Zelditch, M. Zworski, Spacing between phase shifts in a simple scattering problem, Comm. Math. Phys. 204 (1999), no. 3, 709-729.

Department of Mathematics, King's College London, Strand, London, WC2R 2LS, U.K. E-mail address: daniel.bulger@kcl.ac.uk

E-mail address: alexander.pushnitski@kcl.ac.uk 